\title{
Care for Parkinson's Disease Patients in Pakistan: A Call for Help During the COVID-19 Pandemic
}

\author{
Soha Zahid ${ }^{1}$, Sajjad Ali ${ }^{*}$, and Areeb Mohsin ${ }^{1}$ \\ ${ }^{1}$ Department of Medicine, Jinnah Medical and Dental College, Karachi, Sindh, Pakistan \\ 'Department of Medicine, Ziauddin University, Karachi, Sindh, Pakistan
}

Citation: Zahid, S., Ali, S., \& Mohsin, A. (2020). Care for Parkinson's disease patients in Pakistan: A call for help during the COVID-19 pandemic. NeuroRegulation, 7(4), 156-157. https://doi.org/10.15540/nr.7.4.156

*Address correspondence to: Mr. Sajjad Ali, Department of Medicine, Ziauddin University, 4/B, Saharah-e-Ghalib, Block 6, Clifton Karachi-7500, Sindh, Pakistan. Email: sajjad110@live.com

Copyright: (c) 2020. Zahid et al. This is an Open Access article distributed under the terms of the Creative Commons Attribution License (CC-BY).

Parkinson's disease (PD) is an idiopathic disorder of the extrapyramidal system. As the coronavirus disease 2019 (COVID-19) pandemic unfolds, the continuity of essential services for neurodegenerative disorders and similar chronic health problems is a significant concern especially for South Asian regions, including Pakistan. Reportedly, Pakistan has about 450,000 PD patients afflicted with PD, which accounts for about 219 PD patients per 100,000 individuals (Hussain et al., 2017). As COVID-19 is still prevalent throughout the country, it might contribute to a coexisting burden of PD on our healthcare systems.

The neurological effects of COVID-19 are thoroughly explored along with the effect this may have on patients with neurodegenerative disorders. There is existing evidence of SARS-CoV-2 easily linking with the central nervous system (CNS) through hematogenous or axonal path of olfactory neuroepithelium. Reports indicate that SARS-CoV-2 can reach brain cells, adversely affecting the symptomatology of PD patients (Victorino, Guimarães-Marques, Nejm, Scorza, \& Scorza, 2020). A higher proportion of Parkinson's patients experienced new or deteriorating motor $(63 \%)$ and nonmotor (75\%) symptoms during the COVID-19 pandemic (Brown et. al., 2020). Reportedly, as PD escalates during the sixth or seventh decade of life, it can also be correlated with enhanced symptoms of COVID-19. Along with PD, multiple comorbidities are commonly associated, leading to an overall immunocompromised patient who is more prone to show higher severity of COVID-19 symptoms. In
Edited by:

Rex L. Cannon, PhD, SPESA Research Institute, Knoxville, Tennessee, USA

\section{Reviewed by:}

Rex L. Cannon, PhD, SPESA Research Institute, Knoxville, Tennessee, USA

Randall Lyle, PhD, Mount Mercy University, Cedar Rapids, lowa, USA support, studies suggest that the longer the PD duration, the risk of pneumonia increases and so does the need for management through supplemental oxygen, or hospitalization (Brown et. al., 2020). Furthermore, there are secondary potential aggravated effects of the COVID-19 pandemic on PD patients, such as stress, anxiety, and self-isolation, as well as the effects of the lockdown on sustained immobility.

The benefits of exercise for people with PD are well known. Physical exercise helps to reduce the worsening of PD symptoms and related tension, so encouraging domestic workouts such as online Zoom Pilates or dance lessons can be very useful in preserving optimal well-being throughout the pandemic. In addition, a palliative care strategy should be developed to provide relief from physical, mental, and spiritual distress, as the COVID-19 pandemic has definitely intensified the suffering of patients.

In primary and secondary healthcare systems of Pakistan, elective activities were delayed due to age-related measures. This created a hindrance for PD patients in accessing vital medications that can limit their aggravated symptoms. Even though telemedicine services were introduced, it was either not accessible due to compromised financial support or poor internet connectivity (Elbeddini, To, Tayefehchamani, \& Wen, 2020). As COVID-19 cases rose in Pakistan, hospitals were trembling under the patients' weight. If not diagnosed with COVID-19, PD patients reported disrupted medical 
care, limited exercise and social activities, and, in turn, worsened PD motor and nonmotor symptoms. Patients who experienced these interruptions or who underwent self-isolation reported worsening of Parkinson's symptoms.

Disease outbreaks significantly threaten the healthcare system in a developing country like Pakistan. The situation is further compounded by the shortage of basic health services, inadequate health policy, and poor governance. Statistically speaking, the number of individuals living with PD will rise as Pakistan is currently under attack by the second wave of COVID-19, leading to higher financial and social costs. Up until now, no such studies have been conducted in Pakistan on PD patients during this pandemic. However, care should be taken to avoid adding undue stress to individuals living with PD, in the absence of compelling evidence, and at the same time catering to patients suffering from COVID-19.

\section{Author Disclosure}

The authors declared no potential conflicts of interest with respect to the research, authorship, and/or publication of this article.
This research did not receive any specific grant from funding agencies in the public, commercial, or notfor-profit sectors.

\section{References}

Brown, E. G., Chahine, L. M., Goldman, S. M., Korell, M., Mann, E., Kinel, D. R., ... Tanner, C. M. (2020). The effect of the COVID-19 pandemic on people with Parkinson's disease. Journal of Parkinson's Disease, 10(4), 1365-1377. https://doi.org/10.3233/JPD-202249

Elbeddini, A., To, A., Tayefehchamani, Y., \& Wen, C. (2020). Potential impact and challenges associated with Parkinson's disease patient care amidst the COVID-19 global pandemic. Journal of Clinical Movement Disorders, 7(1), 7. https://doi.org/10.1186/s40734-020-00089-4

Hussain, G., Rasul, A., Anwar, H., Sohail, M. U., Kamran, S. K. S., Baig, S. M., Shabbir, A., \& lqbal, J. (2017). Epidemiological data of neurological disorders in Pakistan and neighboring countries: A review. Pakistan Journal of Neurological Sciences, 12(4), 52-70. Retrieved from https://ecommons.aku.edu/pjns /vol12/iss4/12

Victorino, D. B., Guimarães-Marques, M., Nejm, M., Scorza, F. A., \& Scorza, C. A. (2020). COVID-19 and Parkinson's disease: Are we dealing with short-term impacts or something worse? Journal of Parkinson's Disease, 10(3), 899-902. https://doi.org/10.3233/jpd-202073

Received: December 24, 2020

Accepted: December 26, 2020

Published: December 29, 2020 